\title{
Impact of impaired glomerular filtration rate and revascularization strategy on one-year cardiovascular events in acute coronary syndrome: data from Taiwan acute coronary syndrome full spectrum registry
}

Tsung-Hsien Lin ${ }^{1,2}$, Ho-Tsung Hsin ${ }^{3}$, Chun-Li Wang ${ }^{4,5}$, Wen-Ter Lai ${ }^{1,2^{*}}$, Ai-Hsien Li $i^{3}$, Chi-Tai Kuo ${ }^{4,5}$, Juey-Jen Hwang ${ }^{6}$, Fu-Tien Chiang ${ }^{6}$, Shu-Chen Chang ${ }^{7}$, Chee-Jen Chang ${ }^{8}$ and On behalf of Taiwan ACS Full Spectrum Registry Investigators

\begin{abstract}
Background: The optimal revascularization strategy for patients with impaired glomerular filtration rate (IGFR) has not been established in acute coronary syndrome (ACS). We investigated the prognosis and impact of IGFR and invasive strategy on the cardiovascular outcomes in the ACS population.
\end{abstract}

Methods: In a Taiwan national-wide registry, 3093 ACS patients were enrolled. The invasive strategy was defined as patients with ST-elevation ACS (STE-ACS) undergoing primary angioplasty or fibrinolysis or coronary angiography with intent to revascularization performed within 72 hours of symptom onset in non-ST-elevation ACS (NSTE-ACS). IGFR was defined as an estimated GFR of less than $60 \mathrm{ml} / \mathrm{min}$ per $1.73 \mathrm{~m}^{2}$. Primary endpoint was a composite of death, non-fatal myocardial infarction or stroke at one year.

Results: Patients with IGFR ( $n=1226)$ had more comorbidities but received less evidence-based medications during admission than those without IGFR $(n=1867)$. The primary endpoint-free survival rate is lower in the IGFR patients, in the whole, STE-ACS and NSTE-ACS population (all log-rank tests $p<0.01$ ). Cox regression analysis revealed IGFR subjects had higher primary endpoint after adjusting by age, sex, medication at discharge and traditional risk factors (all $p<0.01$ ). Kaplan-Meier curves showed IGFR patients without invasive strategy had the worst outcome in the STE-ACS and NSTE-ACS population (both $p<0.01$ ). The invasive strategies, either with early angiography only or angioplasty, were associated with reduced primary endpoints among IGFR patients in the NSTE-ACS population (both $p \leqq 0.024$ ).

Conclusions: IGFR patients suffering from ACS had poor prognosis and an invasive strategy could improve cardiovascular outcome in the NSTE-ACS population.

Keywords: Acute coronary syndrome, Chronic kidney disease, Invasive, Revascularization, Angiography

\footnotetext{
*Correspondence: wtlai@cc.kmu.edu.tw

'Division of Cardiology, Department of Internal Medicine, Kaohsiung Medical

University Hospital, No.100, Tzyou 1st Road, Kaohsiung 80708, Taiwan

${ }^{2}$ Department of Internal Medicine, Faculty of Medicine, Kaohsiung Medical

University, Kaohsiung, Taiwan

Full list of author information is available at the end of the article
} 


\section{Background}

Chronic kidney disease (CKD) is a global problem [1]. Patients with CKD have higher risk of progression to end-stage renal disease (ESRD) and poor cardiovascular prognosis [2]. Taiwan has been recognized as an epidemic area of kidney disease with the highest incidence and prevalence rates of ESRD in the world [3]. Although the nationwide CKD Preventive Project with multidisciplinary care program has proved its effectiveness in decreasing dialysis incidence, mortality and medical costs, the number of patients with CKD is still growing due to the increasing prevalence of comorbidities, such as hypertension and diabetes, in Taiwan as well as in the worldwide [4]. Therefore, the development of effective treatment strategies is mandatory for such a high cardiovascular risk population.

The presence of endothelial dysfunction, inflammation, dyslipidemia and activation of the renin-angiotensin system are the main mechanisms by which CKD can induce or complicate cardiovascular disease [5]. Therefore, CKD is not only a coronary risk equivalent for ascertainment of coronary risk but also a risk factor for the development and progression of cardiovascular disease (CVD) [6]. Furthermore cardiovascular death is the leading cause of mortality in the CKD population. Among the patients admitted due to acute coronary syndrome (ACS), those with CKD consistently have a poorer prognosis $[7,8]$. Although recent major clinical trials have shown aggressive medication treatment can improve cardiovascular outcome in patients suffering from ACS, only few patients with impaired glomerular filtration rate (IGFR) were enrolled.

In addition to the medical treatment, coronary revascularization such as percutaneous coronary intervention (PCI) is also proven to reduce cardiovascular morbidity and mortality in the ACS patients. However, limited studies investigated the impact of an invasive strategy on clinical endpoints in patients with ACS and IGFR, as many trials of revascularization excluded patients with IGFR [9]. Furthermore, with some evidence support, many physicians hesitate to perform coronary angiography and revascularization in IGFR patients because of the possibility of contrastinduced nephropathy.

The current analysis from Taiwan ACS Full Spectrum Registry therefore focuses on the prognosis and cardiovascular outcomes of invasive strategy in ACS patients with IGFR, including primary angioplasty or fibrinolysis for ST-elevation (STE-ACS) and early invasive strategy (EIS) for non-ST-elevation patients (NSTE-ACS) [10].

\section{Methods}

\section{Study design}

The study was a prospective, national, multicenter, non-interventional, observational design. Patients recruitment, definition of ACS, inclusion and exclusion had been previously described in detail [11]. Patient data, such as baseline characteristics, risk factors, clinical presentation, clinical diagnosis, in-hospital interventions as well as medications prescribed, were collected from admission to discharge. Patients were followed up at months $3,6,9$ and 12 post-discharge and data was collected on medication usage, revascularization strategy as well as clinical events, like death, myocardial infarction, stroke, revascularization and hospitalization. Monitoring for source documentation and accuracy was performed in $5 \%$ of all case report forms at each recruiting site. This study was carried out in accordance with the local regulatory guidelines and international guidelines for Good Epidemiological Practice [12]. Ethics committee approval was obtained at all trial sites including China University Medical Hospital, Taoyuan General Hospital, Wan-Fang Hospital, Show Chwan Memorial Hospital, ChiaYi Christian Hospital, Kuang Tien General Hospital, National Taiwan University Hospital, Cheng Ching Hospital, Sin Lau Hospital The Presbyterian Church of Taiwan, Tainan Municipal Hospital, Mackay Memorial Hospital, E-Da Hospital, Chi-Mei Hospital, Taichung Armed Forces General Hospital, Taipei Tzu Chi General Hospital, Kaohsiung Medical University Chung-Ho Memorial Hospital, Taichung Veterans General Hospital, Pingtung Christian Hospital, Lo-Tung Po-Ai Hospital, Far Eastern Memorial Hospital, National Cheng Kung University Hospital, National Taiwan University Hospital, Yun-lin Branch, Dalin Tzuchi General Hospital, Kee-lung Hospital, Taipei Veterans General Hospital, Cathay General Hospital, Kaohsiung Veterans General Hospital, Taipei Medical University Hospital, Shin Kong Wu Ho-Su Memorial Hospital, Changhua Christian Hospital, National Taiwan University Hospital, Chung Shan Medical University Hospita, Hualien Tzu Chi General Hospital, Mackay Memorial Hospital, Taitung Branch, Linkou Chang Gung Memorial Hospital, Hsin Chu General Hospital, Kaohsiung Chang Gung Memorial Hospital, Tri-Service General Hospital and Cheng-Hsin Hospital. Written informed consent was obtained from each patient.

Invasive strategy was defined as reperfusion done either by primary angioplasty or thrombolysis in STE-ACS or diagnostic coronary angiography (DCA) with intent to revascularization performed within 72 hours of symptom onset as early invasive strategy in NSTE-ACS.

\section{Calculation of kidney function and definition of IGFR}

Creatinine was analyzed by the Jaffe-kinetic method in the central laboratory of each hospital. Baseline creatinine was defined as creatinine measurement at time of presentation. The estimated glomerular filtration rate (eGFR) was calculated using the Chronic Kidney Disease Epidemiology Collaboration equation [13]. IGFR was defined as a eGFR less than $60 \mathrm{ml} / \mathrm{min}$ per $1.73 \mathrm{~m}^{2}$. This range corresponds to 
stage 3 or higher CKD by the National Kidney Foundation's classification scheme and helps identify individuals with clinically significant CKD [14].

\section{Statistical analyses}

All data were expressed as mean \pm standard deviation (SD). For comparability between groups, a chi-square test was used for categorical variables and analysis of variance (ANOVA) was adopted for continuous variables. One-year survival analysis was performed and the time to event was estimated according to the Kaplan-Meier method and compared using the logrank test. Cox regression analysis was conducted to calculate hazard ratio for CVD after adjusting co-variables including age, sex and evidence-based medicines at discharge (aspirin, clopidogrel, ACE inhibitor, angiotensin II receptor blocker, oral b-blocker and statin), dyslipidemia, hypertension, diabetes, smoking and family history. Analyses were conducted as time to first event without double counting of events within analyses involving composite endpoints.

The primary outcome was the composite of death, non-fatal myocardial infarction and non-fatal stroke at one year. The secondary outcome was the composite of death, non-fatal myocardial infarction, non-fatal stroke, re-hospitalization and revascularization at one year. We analyzed the whole, STE-ACS and NSTE-ACS populations separately. Statistical analysis was performed using SAS software version 9.2 (SAS Institute Inc., Cary, NC, USA). All statistical analyses were performed using a level of $<0.05$ with two-sided testing and this was considered as statistically significant.

\section{Results}

\section{Clinical characteristics}

A total of 3183 eligible patients were enrolled between October 2008 and January 2010 at 39 medical centers and regional hospitals in Taiwan [9]. Among them, 3093 (97.1\%) subjects with renal parameters and 12 months outcome data were analyzed in this study and 1631 (52.7\%) patients were STE-ACS. The mean age of the 3093 patients was $63.11 \pm 13.50$ years old and $78.2 \%$ of the patients were males.

Baseline creatinines were $2.74 \pm 2.59$ and $0.94 \pm 0.18 \mathrm{mg} / \mathrm{dl}$ in the IGFR $(\mathrm{n}=1226)$ and non-IGFR $(\mathrm{n}=1867)$ groups, respectively. Compared with the non-IGFR patients, those with IGFR were older, thinner, lower diastolic blood pressure and faster heart rate and had lower percentage of male patients at presentation. Comorbidities, including hypertension, diabetes, previous coronary artery disease (CAD), previous cerebrovascular accident (CVA) and previous heart failure, were more common in IGFR group but percentage of smoking and family history of CAD were lower (Table 1).

\section{Pharmacological management during admission and at discharge}

Medications prescribed during the first 24 hours and at discharge are shown in Figure 1. Class I guidelinerecommended agents including aspirin, clopidogrel, $\beta$-blocker, angiotensin converting enzyme inhibitor and statins were significantly less prescribed during admission and at discharge in patients with IGFR than those without IGFR, with the exception of angiotensin receptor antagonist.

\section{Cardiovascular outcome and IGFR}

During admission, the mortality in IGFR patients was significantly higher $(3.18 \%$ vs $0.59 \%, \mathrm{p}<0.01)$ but risks of in-hospital infarction and stroke were similar compared with non-IGFR subjects. In-hospital acute renal failure happened more frequently in the IGFR than nonIGFR patients $(4.40 \%$ vs. $0.32 \%, \mathrm{p}<0.01)$.

Kaplan-Meier survival analysis showed that IGFR was associated with higher primary and secondary outcome for the whole, STE-ACS and NSTE-ACS populations at one year (all p < 0.01) (Figure 2). Cox regression analysis found the adjusted hazard ratio (HR) of presence of IGFR in the whole, STE-ACS and NSTE-ACS populations were 1.98 (95\% confidence interval (CI): 1.44-2.73), 1.78 (CI: 1.17-2.72) and 2.27 (CI: 1.38-3.74) for the primary endpoint, respectively (all $\mathrm{p}<0.01$ ). For the secondary endpoint, the HR of presence of IGFR in the whole, STE-ACS and NSTE-ACS populations were 1.25 (CI: 1.07-1.45), 1.17 (CI: 0.96-1.44) and 1.33 (CI: 1.06-1.66), respectively.

In the STE-ACS populations the adjusted HRs of the primary and secondary endpoints were 2.30 (95\% CI: 1.44-3.68, p < 0.01) and 1.40 (95\% CI: 1.10-1.77, p < 0.01) in those with eGFR less than 45 compared with those with eGFR more than $60 \mathrm{ml} / \mathrm{min}$. Compared with those with eGFR $>60 \mathrm{ml} / \mathrm{min}$, the adjusted HRs of the primary and secondary endpoints were 1.27 (95\% CI: 0.74-2.19, $\mathrm{p}=0.393)$ and 0.96 (95\% CI: 0.74-1.26, $\mathrm{p}=0.784)$ in those with eGFR between 45 and $60 \mathrm{ml} / \mathrm{min}$. For the NSTE-ACS populations the adjusted HRs of the primary and secondary endpoints were 1.24 (95\% CI: 0.62-2.47, $\mathrm{p}=0.549)$ and 1.01 (95\% CI: 0.74-1.38, $\mathrm{p}=0.933)$ in those with eGFR between 45 and $60 \mathrm{ml} / \mathrm{min}$ compared with those with eGFR more than $60 \mathrm{ml} / \mathrm{min}$. Compared with those with eGFR $>60 \mathrm{ml} / \mathrm{min}$, the adjusted HRs of the primary and secondary endpoints were 3.00 (95\% CI: 1.77-5.07, $\mathrm{p}<0.01$ ) and 1.55 (95\% CI: 1.21-1.98, $\mathrm{p}<0.01$ ) in those with eGFR less than 45 .

\section{Impact of revascularization strategy and IGFR on cardiovascular outcome}

Among 2909 (94.05\%) patients undergoing diagnostic angiography, $81.53 \%$ were done within 72 hours. Fewer IGFR patients received diagnostic angiography $(90.21 \%$ 
Table 1 Baseline characteristics between those with and without IGFR

\begin{tabular}{|c|c|c|c|c|}
\hline Number (\%)/Mean (SD) & $\begin{array}{c}\text { IGFR } \\
(\mathrm{N}=1226) \\
\end{array}$ & $\begin{array}{r}\text { Non-IGFR } \\
(\mathrm{N}=1867) \\
\end{array}$ & $\begin{array}{c}\text { All } \\
(\mathrm{N}=3093) \\
\end{array}$ & $p$ value \\
\hline Sex (male) & $852(69.49 \%)$ & 1568 (83.99\%) & $2420(78.24 \%)$ & $<0.01$ \\
\hline Age (year) & $70.27 \pm 11.71$ & $58.41 \pm 12.50$ & $63.11 \pm 13.50$ & $<0.01$ \\
\hline \multicolumn{5}{|l|}{ Blood pressure $(\mathrm{mmHg})$} \\
\hline SBP & $139.64 \pm 36.36$ & $139.08 \pm 30.19$ & $139.30 \pm 32.75$ & 0.647 \\
\hline DBP & $78.75 \pm 22.11$ & $83.51 \pm 19.84$ & $81.63 \pm 20.89$ & $<0.01$ \\
\hline Heart rate (beat per minute) & $86.26 \pm 25.82$ & $79.66 \pm 19.39$ & $82.27 \pm 22.38$ & $<0.01$ \\
\hline Height (cm) & $162.27 \pm 8.08$ & $165.12 \pm 7.54$ & $164.00 \pm 7.88$ & $<0.01$ \\
\hline Weight (kg) & $65.56 \pm 12.43$ & $70.34 \pm 12.82$ & $68.44 \pm 12.88$ & $<0.01$ \\
\hline Waist circumference & $89.97 \pm 10.01$ & $90.64 \pm 9.31$ & $90.39 \pm 9.57$ & 0.340 \\
\hline Serum creatinine (mg/dL) & $2.74 \pm 2.59$ & $0.94 \pm 0.18$ & $1.65 \pm 1.86$ & $<0.01$ \\
\hline Dyslipidemia & 479 (39.42\%) & 724 (39.14\%) & $1203(39.25 \%)$ & 0.873 \\
\hline Hypertension & $936(77.23 \%)$ & 1019 (55.08\%) & $1955(63.85 \%)$ & $<0.01$ \\
\hline \multicolumn{5}{|l|}{ Diabetes } \\
\hline Treated & $618(50.78 \%)$ & 491 (26.45\%) & 1109 (36.09\%) & $<0.01$ \\
\hline Diet only & $44(7.32 \%)$ & $59(12.37 \%)$ & $103(9.55 \%)$ & $<0.01$ \\
\hline \multicolumn{5}{|l|}{ Smoker } \\
\hline Current & $337(28.13 \%)$ & $948(51.49 \%)$ & $1285(42.28 \%)$ & $<0.01$ \\
\hline Former & $246(20.53 \%)$ & $261(14.18 \%)$ & $507(16.68 \%)$ & \\
\hline Never & $615(51.34 \%)$ & $632(34.33 \%)$ & $1247(41.03 \%)$ & \\
\hline \multicolumn{5}{|l|}{ Family history } \\
\hline Yes & $134(15.51 \%)$ & $394(26.78 \%)$ & $528(22.61 \%)$ & $<0.01$ \\
\hline No & $730(84.49 \%)$ & $1077(73.22 \%)$ & $1807(77.39 \%)$ & \\
\hline Previous coronary artery disease & $394(32.14 \%)$ & $350(18.75 \%)$ & 744 (24.05\%) & $<0.01$ \\
\hline Previous heart failure & $118(9.62 \%)$ & $48(2.57 \%)$ & $166(5.37 \%)$ & $<0.01$ \\
\hline Previous cerebrovascular accident & 169 (13.78\%) & $113(6.05 \%)$ & $282(9.12 \%)$ & $<0.01$ \\
\hline \multicolumn{5}{|l|}{ In-hospital cardiovascular events } \\
\hline Death & $39(3.18 \%)$ & $11(0.59 \%)$ & $50(1.62 \%)$ & $<0.01$ \\
\hline Re-infarction & $13(1.06 \%)$ & $11(0.59 \%)$ & $24(0.78 \%)$ & 0.144 \\
\hline Stroke & $5(0.41 \%)$ & $7(0.37 \%)$ & $12(0.39 \%)$ & 0.886 \\
\hline Acute renal failure & $54(4.40 \%)$ & $6(0.32 \%)$ & $60(1.94 \%)$ & $<0.01$ \\
\hline
\end{tabular}

SBP, systolic blood pressure; DBP, diastolic blood pressure.
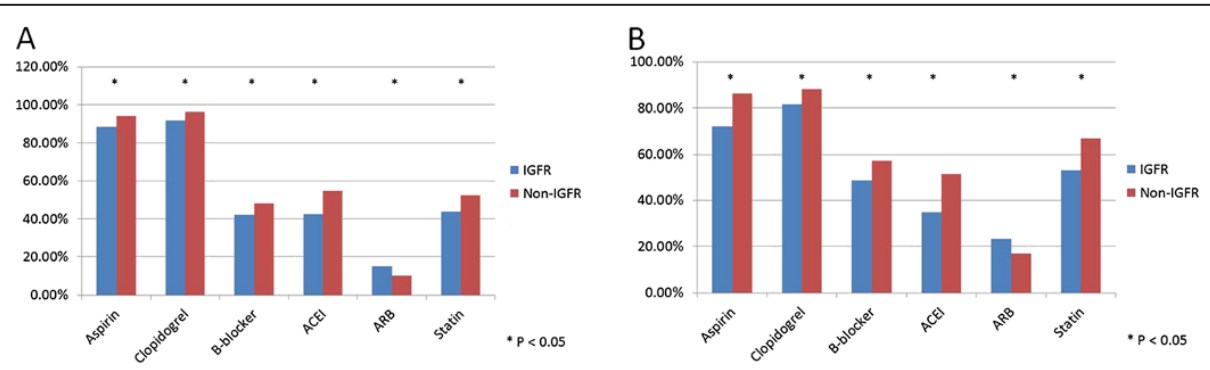

Figure 1 Medication use (A) during the first 24 hours, (B) at discharge between those with and without IGFR. IGFR, impaired glomerular filtration rate; $\mathrm{ACEl}$, angiotensin converting enzyme inhibitor; $\mathrm{ARB}$, angiotensin receptor blocker. 


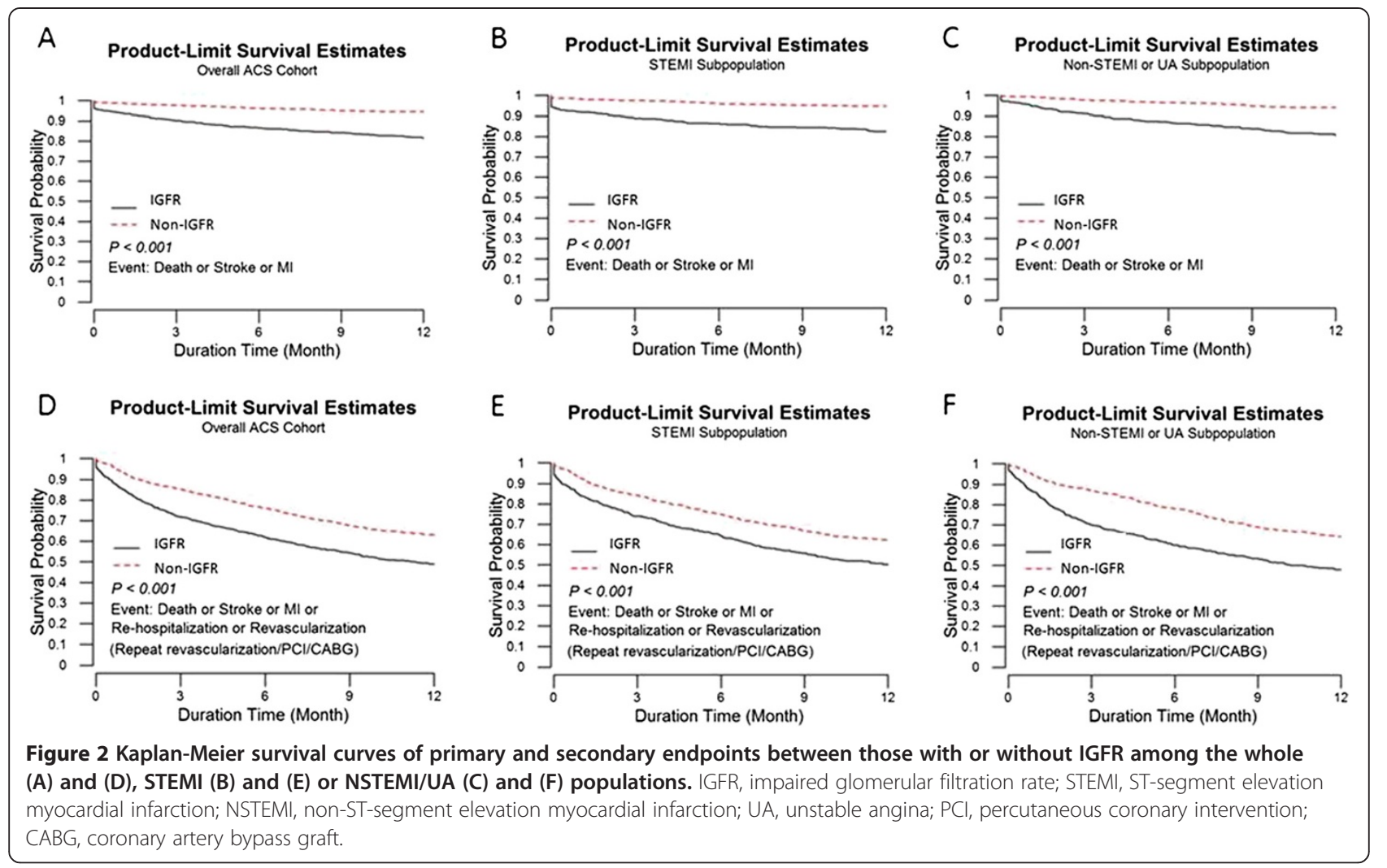

vs. $96.57 \%, \mathrm{p}<0.01)$ with longer time from admission to diagnostic angiography $(51.71 \pm 89.97$ vs. $31.12 \pm 50.04$ hours, $\mathrm{p}<0.01)$ compared with non-IGFR subjects. Overall 2628 (85.31\%) patients received PCI. IGFR patients had lower percentage of received PCI compared with non-IGFR subjects $(79.97 \%$ vs. $88.52 \%, \mathrm{p}<0.01)$. Nevertheless, the percentage of coronary artery bypass grafting (CABG) in IGFR patients was higher $(4.40 \%$ vs. $2.68 \%, \mathrm{p}<0.01)$.

Among the STE-ACS patients, $84.8 \%$ had reperfusion therapy and $96.6 \%$ of them received primary PCI as reperfusion strategy. Lower percentage of IGFR patients received reperfusion therapy compared with non-IGFR subjects $(80.29 \%$ vs. $87.05 \%, \mathrm{p}<0.01)$. However, the percentages of primary PCI were similar between two groups $(97.48 \%$ vs. $97.05 \%, \mathrm{p}=0.653)$.

The Kaplan-Meier survival curves showed significant interaction between revascularization strategy and IGFR on the primary outcome. IGFR patients without invasive strategy had the highest event rate during 1 year followup in the whole, STE-ACS and NSTE-ACS populations (all p < 0.01) (Figure 3).

In IGFR patients Cox regression analysis found invasive strategy did not significantly reduce the primary endpoint (adjusted HR 1.07, 95\% CI: 0.57-1.98; $\mathrm{p}=0.837$ ) in the STE-ACS (Figure 4A). In the NSTE-ACS population, patients receiving either early DCA only or revascularization had lower primary endpoint as compared with those without invasive strategy (adjusted HR 0.53, 95\% CI: 0.30-0.92 and adjusted HR 0.57, 95\% CI: 0.36-0.93; $\mathrm{p}=0.023$ and 0.024 , respectively) (Figure 4B and $\mathrm{C}$ ).

\section{Discussion and conclusion}

There are three major findings in this analysis of Taiwan ACS Full Spectrum cohort study. First, patients with IGFR had more comorbidities but used less guidelinerecommended medicines than those without IGFR. Second, ACS patients with IGFR were more likely to suffer from in-hospital death and 1-year cardiovascular events. Third, invasive strategy might be beneficial for the IGFR subjects in the NSTE-ACS population.

\section{Impaired eGFR and cardiovascular disease}

Impaired eGFR is an important poor predictor of prognosis in those with ACS $[15,16]$. Patients with IGFR have more burden of coronary atherosclerosis in patients with ACS [17]. Although there is no particular reason not to treat IGFR patients just like patients without renal dysfunction, physicians prescribed fewer guidelinerecommended treatments even in the absence of contraindications [18]. Furthermore, poor awareness of impaired renal function and its risk in patients with ACS is a big challenge both for the physicians and patients [19]. As shown in our study, those with IGFR had more comorbidities but received fewer guideline-recommended 

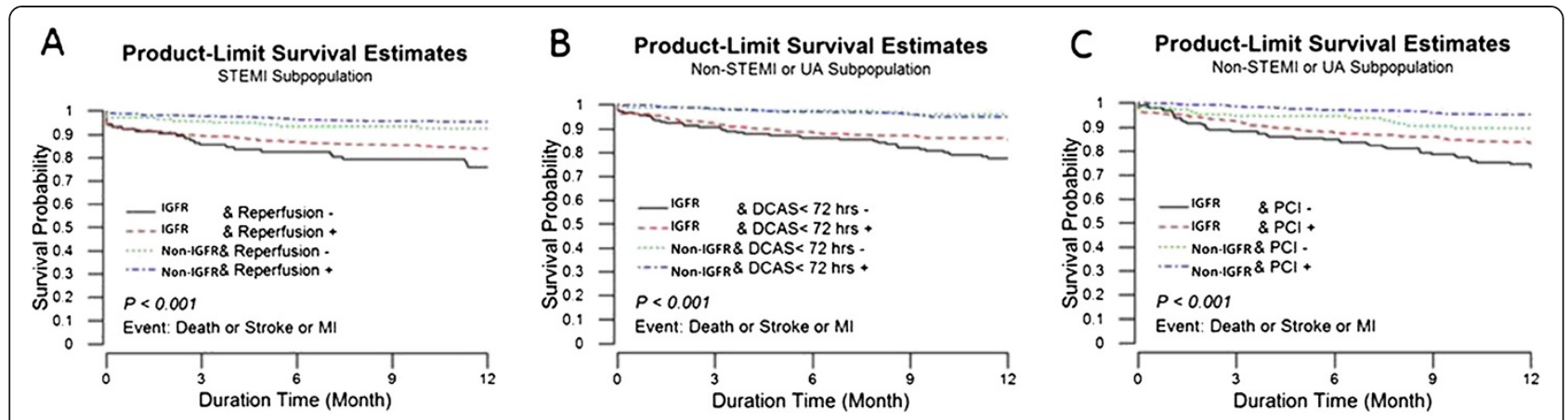

Figure 3 Interaction between invasive strategy and IGFR on the primary outcome. (A). STEMI, (B). NSTEMI or UA and early DCAS, (C). NSTEMI or UA and PCI. IGFR, impaired glomerular filtration rate; STEMI, ST-segment elevation myocardial infarction; NSTEMI, non-ST-segment elevation myocardial infarction; $\mathrm{UA}$, unstable angina; $\mathrm{PCl}$, percutaneous coronary intervention; DCAS, diagnostic coronary angiography strategy.

medications. All of above might contribute to the poor prognosis in the ACS patients with IGFR. Therefore, education and prevention strategies are very important both for physicians and IGFR patients such as the nationwide CKD Preventive Project with multidisciplinary care program in Taiwan [4].

\section{Reperfusion therapy of ST-elevation ACS in IGFR subjects} Underuse of reperfusion therapy is present with all levels of renal dysfunction. Even in patients with mild impairment of eGFR, reperfusion therapy is administered 30\% less frequently. The reason for underuse of reperfusion therapy might be due to limited studies investigating the effect of coronary reperfusion. Furthermore, the use of fibrinolysis therapy might be ineffective and even increase the risk of bleeding in the IGFR subjects $[20,21]$. Therefore, when choosing reperfusion, primary PCI might have greater benefit in patients with IGFR compared with fibrinolytic therapy [22]. As shown in our STE-ACS subgroup, $84.8 \%$ received reperfusion therapy and only $3.4 \%$ of them received fibrinolysis as reperfusion strategy. However, lower percentage of IGFR patients received reperfusion therapy compared with non-IGFR subjects although the percentages of primary PCI were similar between two groups. Whether primary PCI is superior to fibrinolysis in STE-ACS should be further investigated.

\section{Early angiography and revascularization in non-ST-elevation ACS}

Renal dysfunction is present in one-third of patients with NSTE-ACS [23]. Although there are limited studies evaluating the impact of an invasive strategy on clinical endpoints in patients with NSTE-ACS and IGFR, the European society of cardiology guidelines suggests CKD patients are at high risk of further ischaemic events and therefore should be submitted to invasive evaluation and revascularization whenever possible [24]. PCI is also recommended in patients with CKD amenable to revascularization after careful assessment of the risk-benefit ratio in relation to the severity of renal dysfunction [25]. The 2011 ACCF/AHA guideline suggests invasive strategy is reasonable in patients with mild to moderate (stage II-III) CKD [26]. Our results support the recommendations from current guidelines in providing invasive management in ACS patients with IGFR. Although IGFR patients suffering from ACS had poorer prognosis, early diagnostic angiography with intent to revascularization could improve their cardiovascular outcomes in our study.

Influence of IGFR and coronary intervention on ACS subjects Few studies investigated the influence of IGFR on cardiovascular outcomes among ACS population receiving coronary intervention. In patients with STE-ACS receiving primary angioplasty, renal functional impairment based on Cockroft-Gault creatinine clearance or serum creatinine is associated with increased risk of mortality $[27,28]$. In the New York State data, both STE-ACS and NSTE-ACS patients with IGFR and undergoing PCI have significantly worse in-hospital outcomes [29]. In the GRACE registry primary $\mathrm{PCI}$ was associated with lower in-hospital mortality only in patients with normal renal function but no reduction in those with STE-ACS or new-onset of left bundle branch block and renal dysfunction using the MDRD formula [22]. In the SWEDEHEART study early revascularization within 14 days was associated with increased 1-year survival in NSTE-ACS patients with mild to moderate renal impairment, but no association was observed in those with severe or end-stage kidney disease [9]. Hachinohe D. et al. also found early invasive strategy is not beneficial in the severe renal insufficiency population [30]. The present study found IGFR is associated with poor cardiovascular outcome and aggressive strategy given within 72 hours reduced the future occurrence of death, recurrent myocardial infarction and stroke especially in the NSTE-ACS population. 


A
Event
Death. Stroke \& MI
Death. Stroke. MI. Re-hosphilalization
\& Revascularization
All-cause death
Stroke
MI
Re-hosphalization
Revascularization

\section{B}

Event

Death. Stroke \& MI

Death. Stroke, MI, Re-hosptafeation \& Revascularization

All-cause death

Stroke

MI

Re-hospitalzation

Revascularication

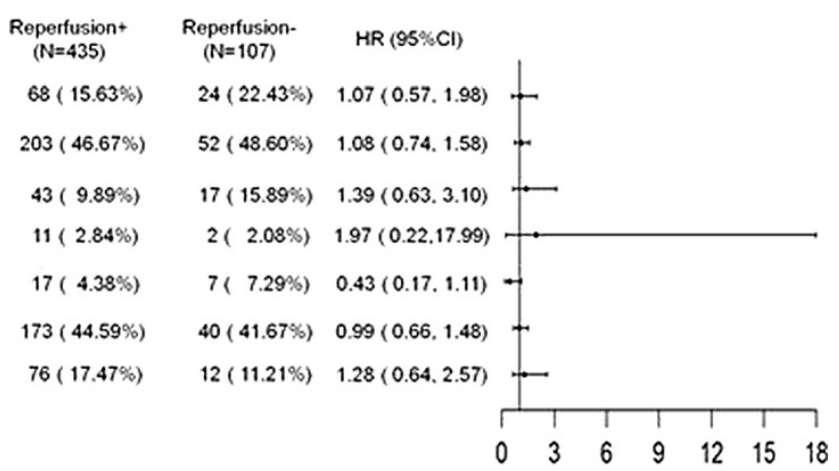

$\begin{array}{lll}\text { DCAS+ } & \text { DCAS. } \\ (\mathrm{N}=355) & \mathrm{N}=190) & H R(95 \% \mathrm{Cl})\end{array}$

$49(13.80 \%) \quad 40(21.05 \%) \quad 0.53(0.30 .0 .92)$

$169(47.61 \%)$

38 ( $10.70 \%)$

$5(1.52 \%)$

$10(3.05 \%)$

148 ( $45.12 \%)$

$49(13.80 \%)$

$99(52.11 \%) \quad 0.84(0.61,1.16)$

$29(15.26 \%) \quad 0.59(0.29,1.18)$

5 ( $2.89 \%) \quad 0.16(0.03,0.97)$

$8(4.62 \%) \quad 0.66(0.20 .2 .16)$

$86(49.71 \%) \quad 0.90(0.65 .1 .27)$

$30(15.79 \%) \quad 0.87(0.49 .1 .55)$

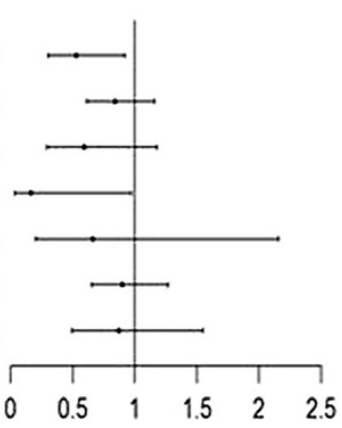

\section{C}

Event

Death. Stroke \& MI

Death. Stroke. MI. Re-hospitalization \& Revascularization

All-cause death

Stroke

MI

Re-hospitalization

Revascularization

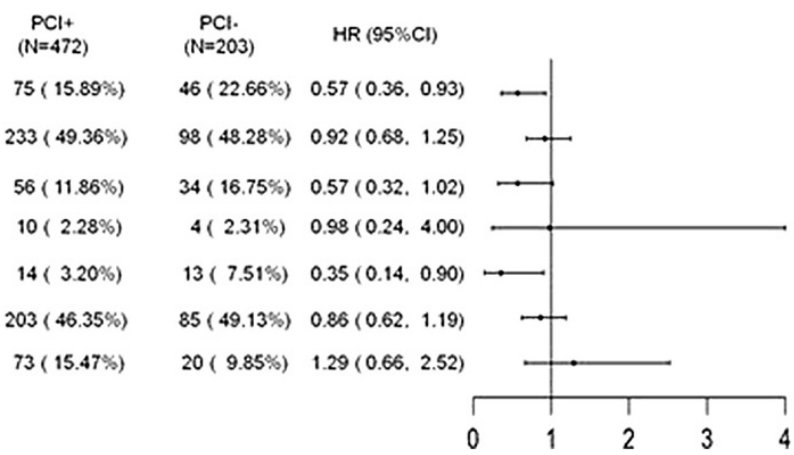

Figure 4 Effect of invasive strategy on cardiovascular events at 12 months in IGFR population. (A). STEMI, (B). NSTEMI or UA and early DCAS, (C). NSTEMI or UA and PCI. IGFR, impaired glomerular filtration rate; STEMI, ST-segment elevation myocardial infarction; NSTEMI, non-ST-segment elevation myocardial infarction; UA, unstable angina; PCl, percutaneous coronary intervention; DCAS, diagnostic coronary angiography strategy.

\section{Limitations}

This study has four main limitations. Firstly, it is a nonrandomized and an observational study. Nonetheless, this study provides valuable real-world data on the current practices across the full spectrum of ACS in a CKD endemic area, which could help to improve the ACS management in this population. Second, the procedural details of revascularization strategy are not available. The lesion characteristics, adjunctive medication and device use might modulate the cardiovascular outcome. Third, the renal endpoint is not routinely collected after discharge in this registry. Although those receiving aggressive revascularization treatment had better cardiovascular outcomes, their renal outcome is unclear. However, Inrig et al. previously reported that ACS patients submitted to angiography or angioplasty had no significant long-term decrease in renal function [31]. The authors suggested that the risk of cardiovascular death for patients with IGFR outweighed the risk of renal function loss or development of chronic dialysis, and angiography or PCI should not be contraindicated in this group. Fourth, there is no universal definition of acute renal failure in 
the registry. The in-hospital acute renal failure was judged at the physicians' discretion.

In conclusion in this real-word registry we found ACS patients with IGFR received fewer evidence-based medicines although having more comorbidities. Furthermore, they were more likely to suffer from in-hospital and 1-year cardiovascular events. Because coronary revascularization could reduce the 1-year cardiovascular events especially in the NSTE-ACS populations, physician should aggressively treat IGFR population after careful assessment of the risk and benefit.

\section{Abbreviations}

CKD: Chronic kidney disease; ESRD: End-stage renal disease; CVD: Cardiovascular disease; ACS: Acute coronary syndrome; eGFR: Estimated glomerular filtration rate; IGFR: Impaired glomerular filtration rate; PCl: Percutaneous coronary intervention; STE-ACS: ST-elevation acute coronary syndrome; EIS: Early invasive strategy (EIS); NSTE-ACS: Non-ST-elevation patients acute coronary syndrome; DCA: Diagnostic coronary angiography; CAD: Coronary artery disease; CVA: Cerebrovascular accident.

\section{Competing interest}

The authors declare that they have no competing interest.

\section{Authors' contributions}

THL, HTH, CLW, WTL, AHL, CTK, JJH and FTC conceived of the study, and participated in its design and coordination. THL drafted the manuscript. SCC and CJC performed the statistical analysis. All authors read and approved the final manuscript.

\section{Acknowledgements}

This study was supported by Sanofi Taiwan Co. Ltd. and Bristol-Myers Squibb (Taiwan) Ltd.

We would like to thank participating physicians and nurses for their contribution in conducting the registry

Principal investigators (by alphabetical order).

Kuan-Chen Chang, China University Medical Hospital; Chia-Lin Chao, Taoyuan General Hospital, Department of Health; Yi-Jen Chen, Wan-Fang Hospital; Chien-Cheng Chen, Show Chwan Memorial Hospital; Cheng-Yun Chen, Chia-Yi Christian Hospital; Chung-Yin Chen, Kuang Tien General Hospital; Fu-Tien Chiang, National Taiwan University Hospital; Shao-Yueh Chiang, Cheng Ching Hospital; Li-Ping Chou, Sin Lau Hospital The Presbyterian Church of Taiwan; Ching-Chang Feng, Tainan Municipal Hospital; Charles Jia-Yin Hou, Mackay Memorial Hospital; Kwan-Li Hsu, E-Da Hospital; Tsuei-Yuan Huang, Chi-Mei Hospital; Gwo-Ping Jong, Taichung Armed Forces General Hospital; Yu-Lin Ko, Taipei Tzu Chi General Hospital; Wen-Ter Lai, Kaohsiung Medical University Chung-Ho Memorial Hospital; Wen-Lieng Lee, Taichung Veterans General Hospital; Chun-I Lee, Pingtung Christian Hospital; Meng-Huan Lei, Lo-Tung Po-Ai Hospital; Ai-Hsien Li, Far Eastern Memorial Hospital; Yi-Heng Li, National Cheng Kung University Hospital; Jou-Wei Lin, National Taiwan University Hospital, Yun-lin Branch; Tin-Kwang Lin, Dalin Tzuchi General Hospital; Jih-Min Lin, Kee-lung Hospital, Department of Health; Shing-Jong Lin, Taipei Veterans General Hospital; Hung-Shun Lo, Cathay General Hospital; Guang-Yuan Mar, Kaohsiung Veterans General Hospital; Chun-Ming Shih, Taipei Medical University Hospital; Kou-Gi Shyu, Shin Kong Wu Ho-Su Memorial Hospital; Cheng-Dao Tsai, Changhua Christian Hospital; Chuen-Den Tseng, National Taiwan University Hospital; Kwo-Chang Ueng, Chung Shan Medical University Hospital; Ji-Hung Wang, Hualien Tzu Chi General Hospital; Kuang-Te Wang, Mackay Memorial Hospital, Taitung Branch; Ming-Shien Wen, Linkou Chang Gung Memorial Hospital; Szu-Chi Wen, Hsin Chu General Hospital, Department of Health; Chiung-Jen Wu, Kaohsiung Chang Gung Memorial Hospital; ShihPeng Yang, Tri-Service General Hospital; Wei-Hsian Yin, Cheng-Hsin Hospital.

\section{Author details}

'Division of Cardiology, Department of Internal Medicine, Kaohsiung Medical University Hospital, No.100, Tzyou 1st Road, Kaohsiung 80708, Taiwan. ${ }^{2}$ Department of Internal Medicine, Faculty of Medicine, Kaohsiung Medical University, Kaohsiung, Taiwan. ${ }^{3}$ Division of Cardiology, Department of
Internal Medicine, Far Eastern Memorial Hospital, Taipei, Taiwan. ${ }^{4}$ Chang Gung University College of Medicine, Taoyuan, Taiwan. ${ }^{5}$ Division of Cardiology, Department of Internal Medicine, Linkou Chang Gung Memorial Hospital, Linkou, Taiwan. ${ }^{6}$ Division of Cardiology, Department of Internal Medicine, National Taiwan University Hospital, Taipei, Taiwan. ${ }^{7}$ Division of Biostatistics, Institute of Public Health, National Yang-Ming University, Taipei, Taiwan. ${ }^{8}$ Graduate Institute of Clinical Medicine, Research Center for Clinical Informatics and Medical Statistics, Chang Gung University, Taoyuan, Taiwan.

Received: 12 January 2014 Accepted: 16 April 2014

Published: 23 April 2014

\section{References}

1. El Nahas AM, Bello AK: Chronic kidney disease: the global challenge. Lancet 2005, 365:331-340.

2. Levey AS, Atkins R, Coresh J, Cohen EP, Collins AJ, Eckardt KU, Nahas ME, Jaber BL, Jadoul M, Levin A, Powe NR, Rossert J, Wheeler DC, Lameire N, Eknoyan G: Chronic kidney disease as a global public health problem: approaches and initiatives - a position statement from kidney disease improving global outcomes. Kidney Int 2007, 72:247-259.

3. USRDS: International comparisons. In United Stated Renal Data System Annual Data Report. Bethesda, MD: The National Institute of Health, National Institute of Diabetes and Digestive and Kidney Disease; 2009:344-355.

4. Hwang SJ, Tsai JC, Chen HC: Epidemiology, impact and preventive care of chronic kidney disease in Taiwan. Nephrology (Carlton) 2010, (Suppl 2):3-9.

5. Schiele F: Renal dysfunction and coronary disease: a high-risk combination. J Nephrol 2009, 22(1):39-45.

6. Brosius FC III, Hostetter TH, Kelepouris E, Mitsnefes MM, Moe SM, Moore MA, Pennathur S, Smith GL, Wilson PW, American Heart Association Kidney and Cardiovascular Disease Council; Council on High Blood Pressure Research; Council on Cardiovascular Disease in the Young; Council on Epidemiology and Prevention; Quality of Care and Outcomes Research Interdisciplinary Working Group: Detection of chronic kidney disease in patients with or at increased risk of cardiovascular disease: a science advisory from the American Heart Association Kidney And Cardiovascular Disease Council; the Councils on High Blood Pressure Research, Cardiovascular Disease in the Young, and Epidemiology and Prevention; and the Quality of Care and Outcomes Research Interdisciplinary Working Group: developed in collaboration with the National Kidney Foundation. Circulation 2006, 114(10):1083-1087.

7. Rodrigues FB, Bruetto RG, Torres US, Otaviano AP, Zanetta DM, Burdmann EA: Effect of kidney disease on acute coronary syndrome. Clin J Am Soc Nephrol 2010, 5(8):1530-1536.

8. Surana SP, Riella LV, Keithi-Reddy SR, Charytan DM, Singh AK: Acute coronary syndrome in ESRD patients. Kidney Int 2009, 75(5):558-562.

9. Szummer K, Lundman P, Jacobson SH, Schön S, Lindbäck J, Stenestrand U, Wallentin $L$, Jernberg T: SWEDEHEART: influence of renal function on the effects of early revascularization in non-ST-elevation myocardial infarction: data from the Swedish web-system for enhancement and development of evidence-based care in heart disease evaluated according to recommended therapies (SWEDEHEART). Circulation 2009, 120:851-858.

10. Chiang FT, Kou-Gi Shyu KG, Wu CJ, Mar GY, Hou Charles JY, Li AH, Wen MS, Lai WT, Lin SJ, Kuo CT, Kuo C, Li YH, Hwang JJ: Predictors of 1-year outcomes in the Taiwan acute coronary syndrome full spectrum registry. J Formos Med Assoc 2013, doi: 10.1016/j.jfma.2013.08.001.

11. Shyu KG, Wu CJ, Mar GY, Hou Charles JY, Li AH, Wen MS, Lai WT, Lin SJ, Kuo CT, Hwang JJ, Chiang FT: Clinical characteristics, management and in-hospital outcomes of patients with acute coronary syndrome - observations from the Taiwan ACS full spectrum registry. Acta Cardiol Sin 2011, 27:135-144.

12. International Epidemiological Association guidelines. http://www.eaweb.org/ index.php?option=com_content\&view=article\&id $=15 \%$ 3Agoodepidemiological-practice-gep\&catid=20\%3Agood-epidemiologicalpracticegep\&ltemid=43\&limitstart=1. Accessed 51128 Jan 2011.

13. Levey AS, Stevens LA, Schmid CH, Zhang YL, Castro AF III, Feldman HI, Kusek JW, Eggers P, Van Lente F, Greene T, Coresh J, CKD-EPI (Chronic Kidney Disease Epidemiology Collaboration): A new equation to estimate glomerular filtration rate. Ann Intern Med 2009, 150(9):604-612.

14. National Kidney Foundation: $\mathrm{K} / \mathrm{DOQ}$ clinical practice guidelines for chronic kidney disease: evaluation, classification, and stratification. Am J Kidney Dis 2002, 39(2 Suppl 1):S1-S266. 
15. AlFaleh HF, Alsuwaida AO, Ullah A, Hersi A, AlHabib KF, AlShahrani A, AlNemer K, AlSaif S, Taraben A, Ahmed WH, Balghith MA, Kashour T: Glomerular filtration rate estimated by the CKD-EPI formula is a powerful predictor of in-hospital adverse clinical outcomes after an acute coronary syndrome. Angiology 2012, 63(2):119-126.

16. Mielniczuk LM, Pfeffer MA, Lewis EF, Blazing MA, de Lemos JA, Shui A, Mohanavelu S, Califf RM, Braunwald E: Estimated glomerular filtration rate, inflammation, and cardiovascular events after an acute coronary syndrome. Am Heart J 2008, 155(4):725-731.

17. Duran M, Uysal OK, Gunebakmaz O, Baran O, Turfan M, Ornek E, Cetin M, Murat SN, Yarlioglues M, Karadeniz M, Kurtul A, Kaya MG: Glomerular filtration rate is associated with burden of coronary atherosclerosis in patients with acute coronary syndrome. Angiology 2014, 65(4):350-356.

18. El-Menyar A, Zubaid M, Sulaiman K, Singh R, Al Thani H, Akbar M, Bulbanat B, Al-Hamdan R, Almahmmed W, Al SJ: In-hospital major clinical outcomes in patients with chronic renal insufficiency presenting with acute coronary syndrome: data from a registry of 8176 patients. Mayo Clin Proc 2010, 85(4):332-340.

19. Babinska M, Chudek J, Ignacy W, Owczarek A, Prochaczek F, Wicek A: Poor awareness of chronic kidney disease in patients with acute coronary syndrome-challenge for cardiologists and nephrologists. Nephrol Dial Transplant 2009, 24(5):1697-1698.

20. Undas A, Nycz K, Pastuszczak M, Stompor T, Zmudka K: The effect of chronic kidney disease on fibrin clot properties in patients with acute coronary syndrome. Blood Coagul Fibrinolysis 2010, 21(6):522-527.

21. Berger $P B$, Best $P J$ : Does kidney function alter the benefit of reperfusion therapy for ST-segment elevation myocardial infarction? JACC CardiovasC Interv 2009, 2(1):34-36.

22. Medi C, Montalescot G, Budaj A, Fox KA, López-Sendón J, FitzGerald G, Brieger DB, GRACE Investigators: Reperfusion in patients with renal dysfunction after presentation with ST-segment elevation or left bundle branch block: GRACE (Global Registry of Acute Coronary Events). JACC Cardiovasc Interv 2009, 2(1):26-33.

23. Goldenberg I, Subirana I, Boyko V, Vila J, Elosua R, Permanyer-Miralda G, Ferreira-González I, Benderly M, Guetta V, Behar S, Marrugat J: Relation between renal function and outcomes in patients with non-ST-segment elevation acute coronary syndrome: real-world data from the European public health outcome research and indicators collection project. Arch Intern Med 2010, 170:888-895.

24. Task Force for Diagnosis and Treatment of Non-ST-Segment Elevation Acute Coronary Syndromes of European Society of Cardiology, Bassand JP, Hamm CW, Ardissino D, Boersma E, Budaj A, Fernández-Avilés F, Fox KA, Hasdai D, Ohman EM, Wallentin L, Wijns W: Guidelines for the diagnosis and treatment of non-ST-segment elevation acute coronary syndromes. Eur Heart J 2007, 28(13):1598-1660.

25. Hamm CW, Bassand JP, Agewall S, Bax J, Boersma E, Bueno H, Caso P, Dudek D, Gielen S, Huber K, Ohman M, Petrie MC, Sonntag F, Uva MS, Storey RF, Wijns W, Zahger D, ESC Committee for Practice Guidelines, Bax J,, Auricchio A, Baumgartner H, Ceconi C, Dean V, Deaton C, Fagard R, Funck-Brentano C, Hasdai D, Hoes A, Knuuti J, Kolh P, et al: ESC Guidelines for the management of acute coronary syndromes in patients presenting without persistent ST-segment elevation: the task force for the management of acute coronary syndromes (ACS) in patients presenting without persistent ST-segment elevation of the European Society of Cardiology (ESC). Eur Heart J 2011, 32(23):2999-3054.

26. Wann LS, Curtis $A B$, January $C T$, Ellenbogen $K A$, Lowe JE, Estes NA III, Page RL, Ezekowitz MD, Slotwiner DJ, Jackman WM, Stevenson WG, Tracy CM, Writing Group Members, Fuster V, Rydén LE, Cannom DS, Le Heuzey JY, Crijns HJ, Lowe JE, Curtis AB, Olsson S, Ellenbogen KA, Prystowsky EN, Halperin JL, Tamargo JL, Kay GN, Wann L, 2006 Writing Committee Members, Jacobs AK, Anderson JL, et al: 2011 ACCF/AHA/HRS focused update on the management of patients with atrial fibrillation (updating the 2006 guideline): a report of the American College of Cardiology Foundation/American Heart Association Task Force on Practice Guidelines. Circulation 2011, 123(1):104-123.

27. Sadeghi HM, Stone GW, Grines CL, Mehran R, Dixon SR, Lansky AJ, Fahy M, Cox DA, Garcia E, Tcheng JE, Griffin JJ, Stuckey TD, Turco M, Carroll JD: Impact of renal insufficiency in patients undergoing primary angioplasty for acute myocardial infarction. Circulation 2003, 108:2769-2775.

28. Yamaguchi J, Kasanuki H, Ishii Y, Yagi M, Ogawa H, Fujii SY, Koganei H, Okada H, Kimura H, Horie T, Haze K, Sumiyoshi T, Honda T, HIJC Study
Group: Prognostic significance of serum creatinine concentration for in-hospital mortality in patients with acute myocardial infarction who underwent successful primary percutaneous coronary intervention (from the Heart Institute of Japan Acute Myocardial Infarction [HIJAMI] Registry). Am J Cardiol 2004, 93:1526-1528.

29. Vasu S, Gruberg L, Brown DL: The impact of advanced chronic kidney disease on in-hospital mortality following percutaneous coronary intervention for acute myocardial infarction. Catheter Cardiovasc Interv 2007, 70:701-705.

30. Hachinohe D, Jeong MH, Saito S, Ahmed K, Hwang SH, Lee MG, Sim DS, Park KH, Kim JH, Hong YJ, Ahn Y, Kang JC, Kim JH, Chae SC, Kim YJ, Hur SH, Seong IW, Hong TJ, Choi D, Cho MC, Kim CJ, Seung KB, Chung WS, Jang YS, Rha SW, Bae JH, Park SJ: Other Korea acute myocardial infarction registry investigators: management of non-ST-segment elevation acute myocardial infarction in patients with chronic kidney disease (from the Korea Acute Myocardial Infarction Registry). Am J Cardiol 2011, 108(2):206-213.

31. Inrig JK, Patel UD, Briley LP, She L, Gillespie BS, Easton JD, Topol EJ, Szczech LA: Mortality, kidney disease and cardiac procedures following acute coronary syndrome. Nephrol Dial Transplant 2008, 23:934-940.

\section{doi:10.1186/1471-2369-15-66}

Cite this article as: Lin et al:: Impact of impaired glomerular filtration rate and revascularization strategy on one-year cardiovascular events in acute coronary syndrome: data from Taiwan acute coronary syndrome full spectrum registry. BMC Nephrology 2014 15:66.

\section{Submit your next manuscript to BioMed Central and take full advantage of:}

- Convenient online submission

- Thorough peer review

- No space constraints or color figure charges

- Immediate publication on acceptance

- Inclusion in PubMed, CAS, Scopus and Google Scholar

- Research which is freely available for redistribution 